\title{
Midterm Electricity Market Clearing Price Forecasting Using Two-Stage Multiple Support Vector Machine
}

\author{
Xing Yan and Nurul A. Chowdhury \\ Department of Electrical Engineering, University of Saskatchewan, Saskatoon, SK, Canada S7N 5A9 \\ Correspondence should be addressed to Xing Yan; xiy201@mail.usask.ca
}

Received 31 May 2014; Revised 14 January 2015; Accepted 15 January 2015

Academic Editor: Chuanwen Jiang

Copyright ( 2015 X. Yan and N. A. Chowdhury. This is an open access article distributed under the Creative Commons Attribution License, which permits unrestricted use, distribution, and reproduction in any medium, provided the original work is properly cited.

\begin{abstract}
Currently, there are many techniques available for short-term forecasting of the electricity market clearing price (MCP), but very little work has been done in the area of midterm forecasting of the electricity MCP. The midterm forecasting of the electricity MCP is essential for maintenance scheduling, planning, bilateral contracting, resources reallocation, and budgeting. A two-stage multiple support vector machine (SVM) based midterm forecasting model of the electricity MCP is proposed in this paper. The first stage is utilized to separate the input data into corresponding price zones by using a single SVM. Then, the second stage is applied utilizing four parallel designed SVMs to forecast the electricity price in four different price zones. Compared to the forecasting model using a single SVM, the proposed model showed improved forecasting accuracy in both peak prices and overall system. PJM interconnection data are used to test the proposed model.
\end{abstract}

\section{Introduction}

Since the generating companies as well as the bulk sellers want to maximize their profits under a deregulated electricity market, offering the appropriate amount of electricity at the right time with the right bidding price is of paramount importance. The forecasting of the electricity price is a prediction of future electricity price based on given forecast electricity demand, temperature, sunshine, fuel cost, precipitation, and other related factors. The electricity market clearing price (MCP) also called the equilibrium price exists when an electricity market is clear of shortage and surplus. Once the electricity MCP is determined, every supplier whose offering price is below or equal to the electricity MCP will be picked up to supply electricity at that hour. To ensure fairness of the market and to avoid market manipulation, the picked up suppliers will be paid at the electricity MCP, not the price they offered.

Currently, in the electricity price forecasting studies, short-term forecasting of the electricity MCP is the most focused research area. It is also commonly known as the 24hour day-ahead electricity price forecasting. Unlike the shortterm forecasting, very little work has been done to forecast electricity MCP on a midterm basis [1-5]. The midterm forecasting of the electricity $\mathrm{MCP}$ focuses on a time frame from one month to six months. It is essential for decision making and midterm planning purposes such as generation plant expansion and maintenance schedule, reallocation of resources, bilateral contracts, and hedging strategies [5]. The midterm forecasting of the electricity MCP is different from the short-term forecasting in many ways. First of all, midterm forecasting by its nature cannot utilize the trend from the immediate past while the short-term forecasting can. Future segment is not contiguous to the immediate past history for which electricity MCP data are available for the midterm forecasting of the electricity MCP. In order to accurately forecast the future electricity MCP with segmented input data, the midterm forecasting model must possess very strong adaptability in handling out-of-sample and segmented data during training phase. Secondly, because of the unavailability of immediate past data, forecasting technique such as time series cannot be utilized in midterm forecasting. In midterm electricity price forecasting, each input data at hour $t$ has to be treated independently from its nearby data such as $t-n$ or $t+n$, for $n$ equals $1,2,3, \ldots$. Moreover, locating and predicating peak prices in the midterm forecasting of the electricity MCP are extremely difficult. As the shortterm forecasting can utilize the trend from the immediate 
past, locating the peak prices in most cases is usually very accurate. The main challenge in short-term forecasting is how accurately the forecasting model can predict the values at each peak price spot. However, in the midterm forecasting of the electricity MCP, because of the unavailability of data from the immediate past, locating the peak prices becomes extremely difficult. As a result, accurately predicting the values at peak price spots becomes even more difficult. The fourth difference is the length of historical data that are needed to train the forecasting model. The short-term forecasting of the electricity price model usually only needs the data of the last couple of days to train the forecasting model. On the other hand, the midterm forecasting model requires one year of historical data in order to train the forecasting model. Finally, only nonlinear regression based forecasting models are capable of midterm forecasting. Linear regression based forecasting models can only be considered as an add-on module in the midterm forecasting.

Before hybrid models utilized in the forecasting of the electricity MCP, autoregressive integrated moving average (ARIMA) [6], wavelet transform [7-9], Monte Carlo simulation [10], bid-based stochastic model [10], time series $[11,12]$, and dynamic regression [13] are the first generation of techniques utilized in the forecasting of the electricity MCP. Artificial neural network (ANN) was later on applied to forecast short-term electricity MCP [2-4, 14-19] because of its flexibility in handling highly nonlinear relationships and relatively easy implementation. PJM interconnection, Australian electricity market, England-Wales pool, and New England ISO [16] are currently utilizing ANN to forecast the electricity MCP.

Support vector machine (SVM) is a new learning method based on structural risk minimization and has gained increased attention in recent electricity price forecasting [20$23]$ studies. The major advantages of SVM over ANN or any other forecasting models are that SVM can avoid problems such as data overfitting, local minimum, and unpredictably large out-of-sample data error while at the same time still achieving better results. SVM is also a very robust forecasting model and will always end up with the same acceptable result regardless of the initial values. On top of that, SVM has less adjustable parameters compared to ANN and is less complicated in control parameter selection. In electricity price forecasting, a traditional SVM can achieve around 3\% [24] improved forecasting accuracy compared to a traditional ANN. Several algorithms including genetic algorithm (GA) [24-27], artificial fish swarm algorithm [28], independent component analysis (ICA) algorithm [29, 30], and rough sets algorithm [31,32] are used to further optimize the training of SVM. Moreover, the least squares support vector machine (LSSVM) was later developed to improve the accuracy of the original SVM $[25,27,33-35]$. Although each method has shown some improvements, the overall system accuracy was still quite low.

The new trend in recent short-term forecasting of the electricity price studies is using hybrid models combining several prediction methods. Hybrid models can compensate the weaknesses of utilizing any individual established method and achieve better overall system results. Torghaban et al. [5] proposed hybrid midterm electricity monthly average price forecasting models combined with SVM/SVM, SVM/NN, NN/SVM, and NN/NN. Swief et al. [23] utilized the principle component analysis (PCA) and $k$ nearest neighbor $(k \mathrm{NN})$ points technique to reduce the number of data entries to the SVM model. Wang and Qin [32] introduced an electricity price prediction model using rough sets and SVM where the rough sets theory was used to simplify the SVM input variables. Xie et al. [33] proposed a hybrid electricity price forecasting model that integrates clustering algorithm with LSSVM. Fan et al. [35] proposed a hybrid forecasting model that includes the Bayesian clustering by dynamics (BCD) and SVM where the BCD classifier is applied to cluster the input data set into several subsets in an unsupervised manner. Zhao et al. [36] forecasted the prediction interval of the electricity price utilizing SVM and nonlinear conditional heteroscedastic forecasting (NCHF) model. They also proposed a hybrid model $[37,38]$ using SVM and probability classifier to predict the price spike occurrence and values. Areekul et al. [39] proposed forecast techniques based on autoregressive integrated moving average (ARIMA) and ANN. Xie et al. [40] proposed a sensitivity analysis of electricity price utilizing SVM and regression model where several price-load elasticity equations are built based on the price pattern data sets classified by SVM classification. A similar work focused on price-load elasticity analysis is done by Wang in [41]. Pousinho et al. [42] proposed a hybrid short-term electricity price forecasting model combined with particle swarm optimization (PSO) and adaptive-network based fuzzy inference system (ANFIS).

A new midterm forecasting model of the electricity MCP utilizing two-stage multiple SVM is proposed in this paper based on the studying of previous works in midterm forecasting of the electricity MCP shown in Table 1 . The proposed model can predict both patterns and values of electricity MCP. A single SVM is first used to separate the input data into corresponding price zones. Then, four parallel designed SVMs are used to forecast the price values inside each separated price zone. The main contributions of this paper include (1) addressing and resolving problems associated with midterm forecasting of the electricity MCP, (2) addressing and resolving the problems associated with using a single nonlinear forecasting model to forecast midterm electricity MCP, such as SVM, and (3) presenting a twostage multiple SVM based forecasting model. Experimental results using historical data from the PJM interconnection system demonstrated that the proposed two-stage multiple SVM forecasting model can improve the price predicting accuracy in both low and peak price zones. The rest of the paper is organized as follows. Two-stage multiple SVM based midterm forecasting model of the electricity MCP is given in Section 2. Case studies are presented in Section 3. Conclusions are included in Section 4.

\section{Two-Stage Multiple SVM Based Midterm Forecasting Model of the Electricity MCP}

2.1. Support Vector Machine. SVM was first introduced by Vladimir Vapnik in 1979 based on the statistical learning and 
TABLE 1: Comparison of previously published midterm forecasting techniques of electricity prices.

\begin{tabular}{|c|c|c|c|}
\hline Authors & Forecasting objectives and techniques & Strengths & Weaknesses \\
\hline Torbaghan et al. [1] & $\begin{array}{l}\text { Midterm forecasting of the monthly } \\
\text { average electricity spot prices using } \\
\text { various techniques }\end{array}$ & $\begin{array}{l}\text { Both single and hybrid models } \\
\text { are tested }\end{array}$ & $\begin{array}{l}\text { Only focused on the monthly } \\
\text { average electricity spot prices } \\
\text { (only } 12 \text { outputs) }\end{array}$ \\
\hline $\begin{array}{l}\text { Yan [2], Yan and } \\
\text { Chowdhury }[3,4]\end{array}$ & $\begin{array}{l}\text { Midterm forecasting of the electricity } \\
\text { hourly MCP utilizing ANN technique }\end{array}$ & Extended training data set & $\begin{array}{l}\text { Poor performance in forecasting } \\
\text { peak prices }\end{array}$ \\
\hline Torghaban et al. [5] & $\begin{array}{l}\text { Midterm forecasting of the monthly } \\
\text { average electricity spot prices utilizing } \\
\text { linear forecasting models }\end{array}$ & $\begin{array}{l}\text { Dummy variables are considered } \\
\text { in the model indicating } \\
\text { seasonality during simulation }\end{array}$ & $\begin{array}{l}\text { Only focused on the monthly } \\
\text { average electricity spot prices } \\
\text { (only } 12 \text { outputs) }\end{array}$ \\
\hline $\begin{array}{l}\text { Yan and } \\
\text { Chowdhury [43] }\end{array}$ & $\begin{array}{l}\text { Midterm forecasting of the electricity } \\
\text { hourly MCP utilizing hybrid LSSVM } \\
\text { and ARMAX techniques }\end{array}$ & $\begin{array}{l}\text { The proposed hybrid model is } \\
\text { capable of adjusting the errors } \\
\text { from the previous module }\end{array}$ & $\begin{array}{l}\text { Limited improvements in } \\
\text { forecasting peak prices }\end{array}$ \\
\hline $\begin{array}{l}\text { Pedregal and } \\
\text { Trapero [44] }\end{array}$ & $\begin{array}{l}\text { Midterm forecasting of the electricity } \\
\text { hourly price utilizing unobserved } \\
\text { component models }\end{array}$ & $\begin{array}{l}\text { Utilization of numbers framed } \\
\text { short-term forecast results to } \\
\text { serve in the midterm forecasting }\end{array}$ & $\begin{array}{l}\text { The proposed model is compared } \\
\text { with an ARIMA model which } \\
\text { has quite weak performance in } \\
\text { midterm forecasting }\end{array}$ \\
\hline González et al. [45] & $\begin{array}{l}\text { Midterm forecasting of electricity hour } \\
\text { price utilizing hybrid approaches } \\
\text { based on the analysis between market } \\
\text { price and marginal costs }\end{array}$ & $\begin{array}{l}\text { Forecast the real price based on } \\
\text { adjusting the prediction values of } \\
\text { the marginal price forecasting }\end{array}$ & $\begin{array}{l}\text { Only price data is involved in the } \\
\text { proposed study and the forecast } \\
\text { of the marginal price is under an } \\
\text { ideal electricity market }\end{array}$ \\
\hline
\end{tabular}

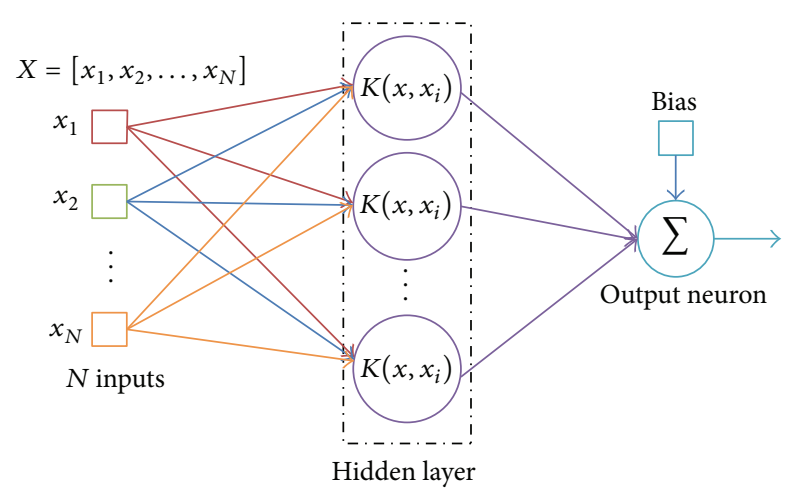

FIGURE 1: SVM architecture [36].

later on developed by Vladimir Vapnik and his coworkers at the AT\&T Bell Laboratories in 1995. After the book An Introduction of Support Vector Machines and Other KernelBased Learning Methods by Nello Cristianini and John ShaweTaylor published in 2000, it started getting more popularity and application in many fields. At the early stage, SVM was only used for classification purposes. Then, the regression computation of nonlinear function was added by solving a convex quadratic optimization problem [46].

A brief SVM architecture is shown in Figure 1. It is very similar to a 3-layer feed-forward ANN. It has the input and output layers containing either single or multiple input/output data. The only difference is inside the hidden layer where kernels replace the hidden neurons. The working principle of a SVM is also different from that of an ANN. A 3-layer feed-forward ANN contains two transfer functions connecting the input layer to the hidden layer and the hidden layer to the output layer. SVM, on the other hand, only has kernels acting like transfer functions inside the hidden layer connecting the input layer and the output layer. Kernels transfer low dimensional input data vector into a much higher dimensional vector (sometime can be infinite) and eventually transfer the highly nonlinear problem inside the input space into a linear problem inside the feature space. After the transformation is completed, optimization algorithms are then applied in order to perform the regression or classification computation. SVM uses a quadratic formulation as optimization algorithm.

Suppose that $\left\{\left(X_{t}, y_{t}\right)\right\}$ for $t=1$ to $N$ is a given set of data, where $X_{t}=\left(x_{t 1}, x_{t 2}, \ldots, x_{t k}\right)$ is the input vector at time $t$ with $k$ elements and $y_{t}$ is the corresponding price data at time $t$ which could be defined as

$$
y_{t}=f\left(X_{t}\right)=\left\langle W, \varphi\left(X_{t}\right)\right\rangle+b
$$

where $\langle$,$\rangle denotes the dot product, W$ is the weight vector, $b$ is the bias, and $\varphi()$ is the mapping function transfers input vector $X_{t}$ into a much higher dimensional feature space (could be infinite). The corresponding optimization problem is then

$$
\begin{aligned}
& \operatorname{minimize} \frac{1}{2}\|W\|^{2}+C \sum_{t=1}^{N}\left(\xi_{t}+\xi_{t}^{*}\right), \\
& \text { subject to } y_{t}-\left\langle W, \varphi\left(X_{t}\right)\right\rangle-b \leq \varepsilon+\xi_{t}, \\
&\left\langle W, \varphi\left(X_{t}\right)\right\rangle+b-y_{t} \leq \varepsilon+\xi_{t}^{*}, \\
& \xi_{t}, \xi_{t}^{*} \geq 0,
\end{aligned}
$$

where $C$ is the regularization constant with regard to the unit cost of errors. $\xi_{t}$ and $\xi_{t}^{*}$ are the slack variables measuring the cost of the errors both above and below the target value in 
the training points. The $\varepsilon$-insensitive loss function with $2 \varepsilon$ bandwidth is defined as

$$
\left|y_{t}-f\left(X_{t}\right)\right|_{\varepsilon}= \begin{cases}0, & \text { if }\left|y_{t}-f\left(X_{t}\right)\right| \leq \varepsilon \\ \left|y_{t}-f\left(X_{t}\right)\right|-\varepsilon, & \text { otherwise. }\end{cases}
$$

When adding the Lagrange multipliers to (2), the problem can be rewritten as

$$
\begin{aligned}
& L\left(W, \xi, \xi^{*}, \alpha, \alpha^{*}, \eta, \eta^{*}\right) \\
& =\frac{1}{2}\|W\|^{2}+C \sum_{t=1}^{N}\left(\xi_{t}+\xi_{t}^{*}\right) \\
& \quad-\sum_{t=1}^{N} \alpha_{t}\left(\varepsilon+\xi_{t}-y_{t}+\left\langle W, \varphi\left(X_{t}\right)\right\rangle+b\right) \\
& -\sum_{t=1}^{N} \alpha_{t}^{*}\left(\varepsilon+\xi_{t}^{*}+y_{t}-\left\langle W, \varphi\left(X_{t}\right)\right\rangle-b\right) \\
& -\sum_{t=1}^{N}\left(\eta_{t} \xi_{t}+\eta_{t}^{*} \xi_{t}^{*}\right),
\end{aligned}
$$

where $\alpha_{t}, \alpha_{t}^{*}, \eta_{t}$, and $\eta_{t}^{*}$ are the Lagrange multipliers. The partial derivates of the Lagrange function with the primal variables $\left(W, b, \xi_{t}\right.$, and $\left.\xi_{t}^{*}\right)$ are then

$$
\begin{aligned}
\frac{\partial L_{\mathrm{SVM}}}{\partial W} & =0 \longrightarrow W=\sum_{t=1}^{N}\left(\alpha_{t}-\alpha_{t}^{*}\right) \varphi\left(X_{t}\right) \\
\frac{\partial L_{\mathrm{SVM}}}{\partial b} & =0 \longrightarrow \sum_{t=1}^{N}\left(\alpha_{t}-\alpha_{t}^{*}\right)=0 \\
\frac{\partial L_{\mathrm{SVM}}}{\partial \xi_{t}} & =0 \longrightarrow C-\alpha_{t}-\eta_{t}=0 \\
\frac{\partial L_{\mathrm{SVM}}}{\partial \xi_{t}^{*}} & =0 \longrightarrow C-\alpha_{t}^{*}-\eta_{t}^{*}=0 .
\end{aligned}
$$

The dual problem can be obtained by substituting the relations from (5):

$$
\begin{gathered}
\operatorname{maximize}-\frac{1}{2} \sum_{t, l=1}^{N}\left(\alpha_{t}-\alpha_{t}^{*}\right)\left(\alpha_{l}-\alpha_{l}^{*}\right)\left\langle\varphi\left(X_{t}\right), \varphi\left(X_{l}\right)\right\rangle \\
-\varepsilon \sum_{t=1}^{N}\left(\alpha_{t}+\alpha_{t}^{*}\right)+\sum_{t=1}^{N} y_{t}\left(\alpha_{t}-\alpha_{t}^{*}\right),
\end{gathered}
$$

subject to $\sum_{t=1}^{N}\left(\alpha_{t}-\alpha_{t}^{*}\right)=0$,

$$
\alpha_{t}, \alpha_{t}^{*} \in[0, C] \text {. }
$$

According to the Karush-Kuhn-Tucker (KKT) conditions, the terms inside the equation containing the Lagrange multipliers will vanish at the optimal solution. This gives the following equations:

$$
\begin{gathered}
\alpha_{t}\left(\varepsilon+\xi_{t}-y_{t}+\left\langle W, \varphi\left(X_{t}\right)\right\rangle+b\right)=0, \\
\alpha_{t}^{*}\left(\varepsilon+\xi_{t}^{*}+y_{t}-\left\langle W, \varphi\left(X_{t}\right)\right\rangle-b\right)=0, \\
\eta_{t} \xi_{t}=\left(C-\alpha_{t}\right) \xi_{t}=0, \\
\eta_{t}^{*} \xi_{t}^{*}=\left(C-\alpha_{t}^{*}\right) \xi_{t}^{*}=0 .
\end{gathered}
$$

The above equations indicate that for all samples whose Lagrange multipliers are equal to zero are considered as nonsupport vectors. Samples with nonzero coefficients are considered as support vectors. Meanwhile, $b$ can be calculated when the slack variables $\xi_{t}$ and $\xi_{t}^{*}$ are equal to zero

$$
b=y_{t}-\left\langle W, \varphi\left(X_{t}\right)\right\rangle \pm \varepsilon \quad \text { for } \alpha_{t}, \alpha_{t}^{*} \in[0, C] .
$$

The final SVM for nonlinear functions can be written as

$$
y_{t}=f\left(X_{t}\right)=\sum_{t=1}^{N}\left(\alpha_{t}-\alpha_{t}^{*}\right)\left\langle\varphi(X), \varphi\left(X_{t}\right)\right\rangle+b .
$$

A modified version of (9) can be expressed as

$$
y_{t}=f\left(X_{t}\right)=\sum_{t=1}^{N}\left(\alpha_{t}-\alpha_{t}^{*}\right) K\left(X, X_{t}\right)+b
$$

where $K\left(X, X_{t}\right)=\left\langle\varphi(X), \varphi\left(X_{t}\right)\right\rangle$ is called the kernel function. Gaussian radial basis kernel (11) is the most powerful one in nonlinear function estimation:

$$
K\left(X, X_{t}\right)=\exp \left(-\frac{\left\|X-X_{t}\right\|^{2}}{2 \sigma^{2}}\right) .
$$

2.2. Price Zones Analysis. One of the special characteristics of electricity is that it has to be produced as used. Taking PJM interconnected electricity market as an example, electricity price can vary from as low as $-\$ 10 / \mathrm{MWh}$ to as high as $\$ 748 / \mathrm{MWh}[30]$. The relationship between supply and demand under normal deregulated market is no longer the only dominant principle determining the electricity price. Other factors such as business competing strategy and unethical business behaviour are heavily involved and cannot be efficiently simulated. Therefore, the accuracy of the forecasted electricity MCP in the peak price zone is always very low. Although electricity MCP is very volatile, it is still normally distributed along its average value [20-22]. In the proposed work, the electricity MCP is separated into four price zones: low, medium, high, and peak price zones. Let $\mu$ and $\sigma$ be the mean and standard deviation of the monthly historical electricity MCP; the four price zones of each month are determined based on the four criteria listed below:

$$
\begin{aligned}
& \text { low: } \mathrm{MCP}<\mu-\sigma \text {; } \\
& \text { medium: } \mu-\sigma \leq \mathrm{MCP}<\mu+0.5 \sigma \\
& \text { high: } \mu+0.5 \sigma \leq \mathrm{MCP}<\mu+1.5 \sigma \\
& \text { peak: } \mu+1.5 \sigma \leq \mathrm{MCP} \text {. }
\end{aligned}
$$


About $15 \%-25 \%$ of the price is in the low price zone. $50 \%-60 \%$ of the price is in the medium price zone. $15 \%$ $-20 \%$ of the price is in the high price zone. Finally, the last $5 \%-10 \%$ of the price will be in the peak price zone. The standard deviation multipliers are chosen based on the price characteristics of the PJM interconnected electricity market and forecasting model parameter optimization. They can be modified when applying in a different electricity market. Inside the medium price zone, most generating companies are participating in supplying electricity. Every participating generating unit is running at its optimal output. There is plenty of reserved energy available in the market which can be supplied immediately. Congestion is low in most areas and congestion cost is limited. At this moment, the market is at its stable state. Sudden shortage or surplus can be easily recovered or offset by other generating companies in the same and nearby jurisdiction. Supply and demand elasticity plays a major role and electricity MCP is mainly determined by the demand and fuel cost. Inside the low price zone, the profit margin is small and, therefore, only companies with low production cost and mandatory all-time running power units such as nuclear power plants are participating. Sudden shortage or surplus could cause price spikes because they cannot be recovered or offset as easily as in the medium price zone. In the peak price zone, most power plants are running at their peak capacity. Transmission lines are under extreme load resulting in extremely high congestion cost in some areas. System reserved energy is very low and generating companies expect to take advantages and make huge profits from it. Electricity MCP is mainly determined by business competing strategy and even unethical business behaviour in the peak price zone. The high price zone is between the medium and peak price zones and, therefore, shows characteristics of both price zones. The supply and demand relationship and business competing strategy both dominate the final electricity MCP inside the high price zone.

\subsection{Two-Stage Multiple SVM Forecasting Model Architecture.}

The architecture of the proposed two-stage multiple SVM based midterm forecasting model of the electricity MCP is shown in Figure 2. It contains four layers: (1) input layer, (2) input data distribution layer, (3) SVM price prediction layer, and (4) output layer.

Inside the input layer, there will be input data such as electricity hourly demand, electricity daily peak demand, and daily natural gas price. Data selection and preprocessing will be explained in detail in Section 2.4. After all the data are preprocessed inside the input layer, a single SVM is first utilized to predict the initial values of the electricity prices. These price values are then separated into four predefined price zones: low, medium, high, and peak. The input data distribution layer can significantly reduce the range each price prediction SVM shall cover and improve the overall system accuracy when forecasting the midterm electricity MCP. After the input data distribution is completed, the SVM prediction module is utilized to forecast the midterm electricity MCP. There are four parallel connected SVMs inside the price prediction module to forecast the electricity MCP in four different price zones. Based on the different

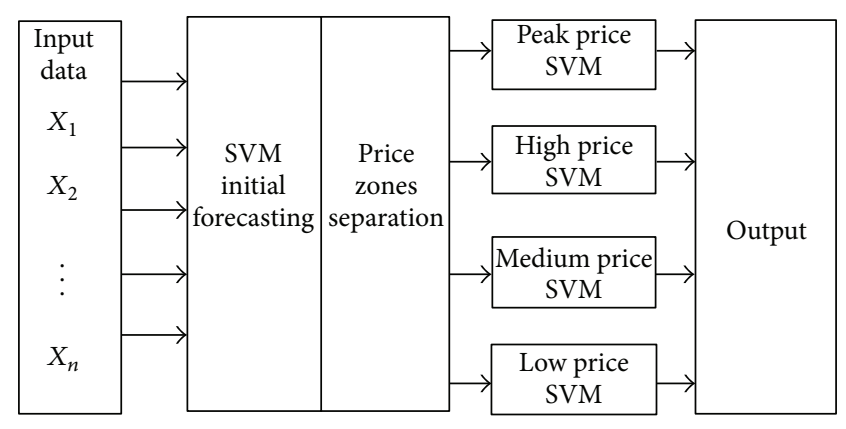

FIGURE 2: Two-stage multiple SVM forecasting model architecture.

range of input data, each price prediction SVM optimizes its own control parameters during the training process. These different control parameters give each price prediction SVM the ability to capture the characteristics between input and output data within each price zone. Inside the output layer, four SVMs predicted electricity price values are combined to form the final forecast electricity MCP. Input data distribution module will assign each input data with serial numbers that will be used to regroup all forecast price values from the SVM price prediction layer inside the output layer. The training input data for both modules are from the input layer in Figure 2. The target data for both modules are the historical electricity MCP. Cross-validation technique is applied in data distribution and price prediction modules for the optimization of the control parameters.

2.4. Data Collection and Preprocessing. Electricity MCP can be forecasted by evaluating a variety of elements [31] such as electricity demand, supply, natural gas price, coal price, hydrocapacity, weather, and temperature. An ideal forecasting model of the electricity MCP should include all the possible elements that affect the final electricity MCP. In reality, however, it is impossible and unnecessary to do so. Since weather conditions including daily temperature are already considered in load forecasting, they do not have to be included in MCP forecasting process. Other elements such as business strategy and unethical competition behaviours cannot be easily represented mathematically. Finally, the current failure or operating status of a generator in many deregulated electric market is confidential information. Due to the factors mentioned above, a data selection process is presented in Figure 3 using cross-validation technique to finalize the input data that were taken into account for the proposed work.

The data selection process starts with only the default input data (hourly electricity demand) inside the forecasting model. Then, the next element inside the potential input data is added to the initial forecasting model to test whether the added element improves the forecasting accuracy or not. Tested element will be added to the forecasting model if it improves the forecasting accuracy. Otherwise, the forecasting model maintains its current input data and the next element inside the potential input data will be tested. Due to the fact that sometimes adding more than one element at a time 


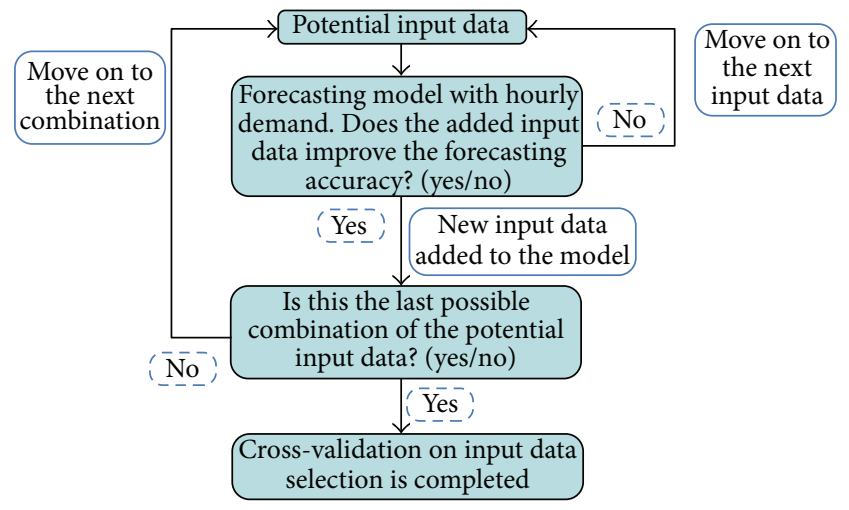

FIGURE 3: Data selection process.

will achieve better forecasting results, different combinations of elements will also be tested once all the elements have been tested one at a time. By applying the data selection process shown in Figure 3, it will guarantee to test not only every single element but also every possible combination of elements that would lead to the optimized forecasting results. The final selected input data for the proposed twostage multiple SVM forecasting model at each hour $t$ include (1) electricity hourly demand at hour $t$, (2) electricity daily peak demand, (3) electricity monthly average demand, (4) daily price of natural gas, (5) previous year's monthly average electricity MCP, (6) month (1-12), and (7) hour of the day (1$24)$. The target data at hour $t$ is the electricity MCP at hour $t$. Moreover, one year is the most optimized length of historical data to train the forecasting model based on the previous published works [1-3] regarding the selection of training data for midterm forecasting of the electricity MCP.

Part of the training data is separated from the rest of the training data and served as the so-called the testing data. The testing data are used to optimize the control parameters of the proposed forecasting model and are treated unseen from the rest of the training data. For the rest of this paper, the remaining part of the training data is called the actual training data. In the proposed work, data from January 1 , 2009, to December 31, 2009, excluding June 2009, are selected as the actual training data and can be viewed as a matrix with $8040(24 \times 335)$ rows and 8 columns. The 8040 rows are the number of hours from hour 1 (1:00 a.m.) on January 1, 2009, to hour 24 (12:00 a.m.) on December 31, 2009, excluding June 2009. The first 7 columns are the 7 input elements mentioned before. The last column is the training target data, electricity MCP from hour 1 (1:00 a.m.) on January 1, 2009, to hour 24 (12:00 a.m.) on December 31, 2009, excluding June 2009. Because the proposed two-stage multiple SVM based midterm forecasting model of the electricity MCP is designed to predict the 720 hourly electricity MCPs in June 2010 , selecting data from hour 1 (1:00 a.m.) on June 1, 2009, to hour 24 (12:00 a.m.) on June 30, 2009, as the testing data could create the best scenarios on daily demand pattern, daily price pattern, weather, sunshine, and precipitation during training process to optimize the control parameters. For the same reason, if the proposed model is utilized to forecast the 744 hourly electricity MCPs in May 2010, data in May 2009 will be selected as the testing data during training process. The testing data contain a matrix with 720 rows $(24 \times 30)$ and 8 columns. The first 7 columns are the testing input data and the last column is the testing target data.

Once the training procedure is done, the proposed twostage multiple SVM forecasting model is used to forecast the midterm electricity MCP of every hour in June 2010 using historical data from January 2009 to December 2009. This work is based on the assumption that all forecasting input data have already been accurately predicted to limit the affects from inaccurate input data. The forecast output contains $720(24 \times 30)$ hourly electricity MCP which will insure the comprehensiveness of the test sample. The forecasting input data could be viewed as a matrix with $720(24 \times 30)$ rows and 8 columns. The 720 rows are the number of hours from hour 1 (1:00 a.m.) on June 1, 2010, to hour 24 (12:00 a.m.) on June 30, 2010. The first 7 columns are the forecasting input data and the last column is the electricity MCP from hour 1 (1:00 a.m.) on June 1, 2010, to hour 24 (12:00 a.m.) on June 30, 2010.

In order to improve the regression accuracy and avoid the dominance of some extremely large values inside the data set, data preprocessing is needed in machine learning. For electricity MCP forecasting, the most common and recommended data preprocessing algorithms are the normalization. Normalization converts each datum inside its corresponding element into a value between -1 and +1 (sometimes between 0 and 1) with respect to each element's maximum and minimum value. Depending on the calculation, the maximum and minimum values can be either global or local. Suppose that $X_{t}=\left(x_{t 1}, x_{t 2}, \ldots, x_{t k}\right)$ is a given set of vectors at time $t$ with $k$ multiple elements for $t=1$ to $N$. The normalization for datum $x_{t k}$ could be defined as

$$
\widehat{x}_{t k}=\frac{x_{t k}-\left(x_{\mathrm{MAX} k}+x_{\mathrm{MINk}}\right) / 2}{x_{\mathrm{MAXk}}-\left(x_{\mathrm{MAXk}}+x_{\mathrm{MINk}}\right) / 2},
$$

where $x_{\mathrm{MAX} k}$ and $x_{\mathrm{MIN} k}$ are the global or local maximum and minimum values inside the $k$ th element. The normalization on forecasting input data is performed using each element's corresponding global or local maximum and minimum values during the training process. The target data remain unchanged as there is only the electricity MCP data. The designed two-stage multiple SVM based midterm forecasting model of the electricity MCP can utilize different dimensions of input data for forecasting accuracy optimization. The full dimension of the input data is 7 as mentioned before. Recall the data selection process from Figure 3 and, using mean absolute error for the performance evaluation, the optimized final dimensions of input data for the proposed multiple SVM are 7 for the initial prediction SVM, 5 for the low price SVM, 7 for the medium price SVM, 7 for the high price SVM, and 7 for the peak price SVM.

2.5. Performance Evaluation. Mean absolute error (MAE), mean absolute percentage error (MAPE), and mean square root error (MSRE) are the three most widely used measurements for performance evaluation in forecasting electricity price values. Given $N$ historical electricity MCP data, $y_{t}$, and 
the corresponding forecasted price data $\widehat{y}_{t}$ for $t=1$ to $N$, MAE, MAPE, and MSRE are defined as

$$
\begin{aligned}
\text { MAE } & =\frac{1}{N} \sum_{t=1}^{N}\left|y_{t}-\hat{y}_{t}\right|, \\
\text { MAPE } & =\frac{1}{N} \sum_{t=1}^{N}\left|\frac{y_{t}-\hat{y}_{t}}{y_{t}}\right| \times 100 \%, \\
\text { MSRE } & =\sqrt{\frac{1}{N} \sum_{t=1}^{N}\left(y_{t}-\widehat{y}_{t}\right)^{2} .}
\end{aligned}
$$

During the training process, two SVM control parameters, the coefficient of the Gaussian radial basis kernel $\sigma$ and the regularization constant $\gamma$, are set at an initial value and updated using testing data during each iteration until the MAE of the SVM reaches its global minimum. Once all control parameters are determined, forecasting input data can be applied to forecast the midterm electricity MCP.

\section{Case Studies}

3.1. PJM Interconnected Electric Market. PJM interconnected electric market is the largest interconnected system in the world. It includes all or most of Delaware, District of Columbia, Maryland, New Jersey, Ohio, Pennsylvania, Virginia, and West Virginia. Parts of Indiana, Illinois, Kentucky, Michigan, North Carolina, and Tennessee are also included in the PJM interconnected electric market. The market serves more than 61 million people in the United States. As of December 31, 2012, it had an installed generating capacity of 183,604 megawatts and more than 800 participating members. The market covered 243,417 square miles of territory containing 62,556 miles of transmission lines and more than 1,376 electric generators [47]. Coal (74\%) and natural gas $(22 \%)$ are the two major types of fuel in the market. Locational marginal pricing (LMP) is used to reflect the value of the energy at the specific location and time it is delivered. The market consists of day-ahead and real-time markets. The day-ahead market is a forward market in which hourly LMP is calculated for the next operating day based on generation offers, demand bids, and scheduled bilateral transactions. The real-time market is a spot market in which current LMP is calculated at five-minute intervals based on actual grid operating conditions. PJM settles transactions hourly and issues invoices to market participants monthly [48]. The LMP inside the PJM interconnected electric market is used as electricity MCP in this work. Historical data from the dayahead market of 2008 and 2009 are used in this work to forecast the electricity MCP in June 2010 and are available at [47].

3.2. Experimental Results. The testing results using both single SVM and the proposed two-stage multiple SVM are shown in Figure 4. Data from January 2009 to December 2009 excluding June 2009 are used as the actual training data and data in June 2009 are used as the testing data.

It can be seen that the major contribution of utilizing a two-stage multiple SVM based forecasting model is the

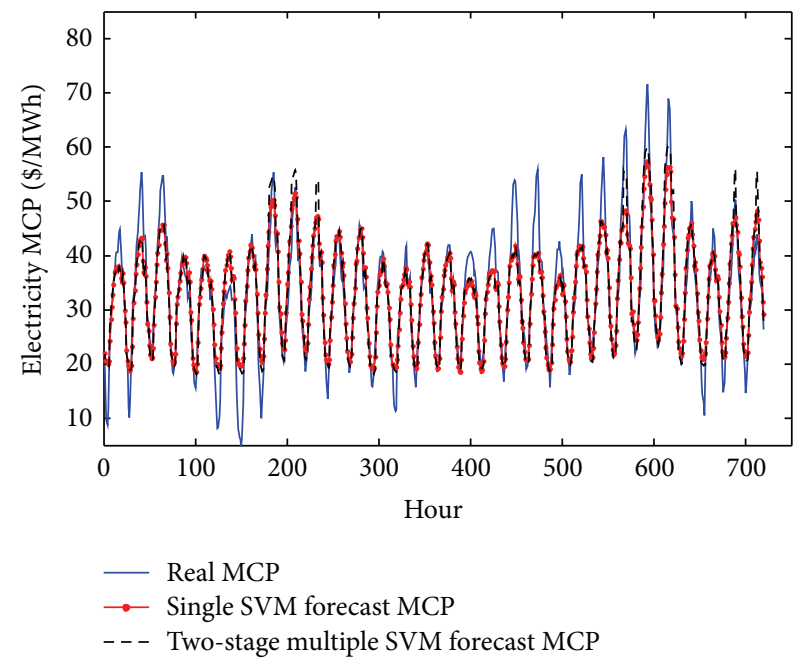

FIGURE 4: Forecasted electricity MCP in June 2009 using both single and two-stage multiple SVM.

TABLE 2: Performance evaluation results of MAE between the single and the two-stage multiple SVM in June 2009.

\begin{tabular}{lccccc}
\hline MAE & Low & Medium & High & Peak & System \\
\hline Single & 4.0094 & 1.8941 & 3.0837 & 8.2403 & 2.9743 \\
Multiple & 3.1985 & 1.8678 & 3.8336 & 5.7636 & 2.9625 \\
PRIM & $20.22 \%$ & $1.39 \%$ & $-24.32 \%$ & $30.06 \%$ & $0.40 \%$ \\
\hline
\end{tabular}

TABLE 3: Performance evaluation results of MAPE between the single and the two-stage multiple SVM in June 2009.

\begin{tabular}{lccccc}
\hline MAPE & Low & Medium & High & Peak & System \\
\hline Single & 35.9821 & 6.1526 & 7.2207 & 14.5750 & 11.7491 \\
Multiple & 24.9639 & 5.6042 & 8.7352 & 11.2517 & 11.4083 \\
PRIM & $30.62 \%$ & $8.91 \%$ & $-20.97 \%$ & $22.80 \%$ & $2.90 \%$ \\
\hline
\end{tabular}

improved forecasting results in the low and peak price zones. The regression computation performance evaluations are performed using MAE, MAPE, and MSRE analyses. The performance numbers are shown in Tables 2, 3, and 4. Percentage improvement (PRIM) is used to compare the regression computation performance between the single SVM model and the proposed two-stage multiple SVM model. The PRIM is calculated as

$$
\begin{aligned}
& \text { PRIM }=((\text { single_MAE } / \text { MSRE/MAPE } \\
& \text { - multiple_MAE/MSRE/MAPE) } \\
& \left.\cdot(\text { single_MAE/MSRE/MAPE })^{-1}\right) \times 100 \% \text {. }
\end{aligned}
$$

Positive PRIM values indicate that the two-stage multiple SVM model is better than the single SVM model in this price zone. Negative PRIM values indicate the opposite.

According to all three tables, MAE, MAPE, and MSRE values show that the two-stage multiple SVM model is more accurate than the single SVM model by more than at least $20 \%$ in the low and peak price zones. However, the results 
TABLE 4: Performance evaluation results of MSRE between the single and the two-stage multiple SVM in June 2009.

\begin{tabular}{lccccc}
\hline MSRE & Low & Medium & High & Peak & System \\
\hline Single & 0.5200 & 0.1191 & 0.2933 & 1.2738 & 0.1564 \\
Multiple & 0.3381 & 0.1408 & 0.3590 & 1.0060 & 0.1536 \\
PRIM & $34.98 \%$ & $-18.22 \%$ & $-22.40 \%$ & $21.02 \%$ & $1.79 \%$ \\
\hline
\end{tabular}

TABLE 5: Performance evaluation results of MAE between the single and the two-stage multiple SVM in June 2010.

\begin{tabular}{lccccc}
\hline MAE & Low & Medium & High & Peak & System \\
\hline Single & 5.7523 & 6.9711 & 5.0316 & 15.9496 & 7.2523 \\
Multiple & 2.7764 & 7.5398 & 7.6556 & 11.1233 & 6.8106 \\
PRIM & $51.73 \%$ & $-8.16 \%$ & $-52.15 \%$ & $30.26 \%$ & $6.09 \%$ \\
\hline
\end{tabular}

are opposite in the high price zones. This is caused by the characteristics of the high price zone as it is between the medium and peak price zones. The supply and demand relationship and business competing strategy both dominate the final electricity MCP inside the high price zone. It is very difficult for a single SVM to simulate these two very different characteristics at the same time. Significantly reduced data caused by the SVM classification process is also another reason; MAE, MAPE, and MSRE are low in the high price zone. As the MSRE values in the medium price zones are opposite compared to the MAE and MAPE values, it is hard to say which model has the better performance in the medium price zone. Usually, the conclusions obtained from the performance numbers of MAE, MAPE, and MSRE should be identical. However, in special cases, the conclusions would differ from each other due to the different distribution of results. For instance, suppose that the forecasting errors from the single SVM and two-stage multiple SVM are $(6,6)$ and (1, 10 ) and the forecasting percentage errors from the single SVM and two-stage multiple SVM are $(6 \%, 6 \%)$ and $(1 \%, 10 \%)$. The size of the data set is only 2 . Therefore, the MAE of the single SVM and the two-stage multiple SVM are $(6+6) / 2=$ 6 and $(1+10) / 2=5.5$. According to the MAE values, the twostage multiple SVM is better than the single SVM. The same conclusions apply to the MAPE evaluation where the MAPEs of the single SVM and the two-stage multiple SVM are $(6 \%$ $+6 \%) / 2=6 \%$ and $(1 \%+10 \%) / 2=5.5 \%$. However, when comparing the MSRE values, the conclusion is different. The MSREs of the single SVM and the two-stage multiple SVM are $\left(6^{2}+6^{2}\right)^{0.5} / 2=4.243$ and $\left(1^{2}+10^{2}\right)^{0.5} / 2=5.025$. According to the MSRE values, the single SVM is better than the twostage multiple SVM. Under special situation like this, when the conclusions obtained by the MAE, MAPE, and MSRE values do not agree with each other in certain price zones (e.g., the medium zone), because all the control parameters are determined based on the global minimal MAE values, we will use the MAE values to determine which forecasting model is better.

As the testing results supported the fact that the twostage multiple SVM is better than the single SVM in midterm forecasting of the electricity MCP, the proposed forecasting model is used to forecast the midterm hourly electricity
TABLE 6: Performance evaluation results of MAPE between the single and the two-stage multiple SVM in June 2010.

\begin{tabular}{lccccc}
\hline MAPE & Low & Medium & High & Peak & System \\
\hline Single & 21.0094 & 16.3663 & 7.5146 & 16.7736 & 15.6454 \\
Multiple & 10.2069 & 18.9028 & 12.4708 & 12.0534 & 14.6567 \\
PRIM & $51.42 \%$ & $-15.50 \%$ & $-65.95 \%$ & $28.14 \%$ & $6.32 \%$ \\
\hline
\end{tabular}

TABLE 7: Performance evaluation results of MSRE between the single and the two-stage multiple SVM in June 2010.

\begin{tabular}{lccccc}
\hline MSRE & Low & Medium & High & Peak & System \\
\hline Single & 0.4572 & 0.3350 & 0.5299 & 2.6890 & 0.3316 \\
Multiple & 0.2569 & 0.4872 & 0.6411 & 1.7935 & 0.3126 \\
PRIM & $43.81 \%$ & $-45.43 \%$ & $-20.99 \%$ & $33.30 \%$ & $5.73 \%$ \\
\hline
\end{tabular}

MCP in June 2010 using historical data from January 2009 to December 2009. Figure 5 shows the forecast electricity hourly MCP in June 2010 using both single and the two-stage multiple SVM. The final midterm forecasting results of the electricity hourly MCP are obtained from the combination of predicted price from four price prediction SVMs. Detailed evaluation results of MAE, MAPE, and MSRE are shown in Tables 5, 6, and 7. The two-stage multiple SVM forecasting model shows an average 6\% improvement on the system MAE, MAPE, and MSRE evaluations. The negative PRIM values in the high price zone are expected while the negative PRIM values in the medium price zone indicate that there is misdistribution during the forecast process. The forecasting results of the proposed multiple SVM model are also compared with other machine learning methods [43] shown in Table 8. Although the proposed two-stage multiple SVM is only slightly better in forecasting accuracy compared with other midterm electricity MCP forecasting methods such as the hybrid LSSVM and ARMAX during the forecasting process, the proposed two-stage multiple SVM model is way more flexible in adjusting input data in any SVM and therefore has much higher potential to improve in future research than other midterm electricity price forecasting methods. All of the study cases were run on a PC with $4 \mathrm{~GB}$ of RAM and a $2.4 \mathrm{GHz}$ processor. MATLAB 7.7 is used. The average forecasting time is less than 1 minute.

3.3. Discussions. The SVM intends to lose the top and the bottom peak values during training process because most of those values are considered as nonsupport vectors when utilizing the $\varepsilon$-insensitive loss function with $2 \varepsilon$ bandwidth. Only the support vectors are used to create the $2 \varepsilon$ tube and only the values outside the $2 \varepsilon$ tube are considered during simulation. As mentioned before, the electricity MCP in the peak price zone is mainly determined by business competing strategy and even unethical business behaviour and those elements cannot be easily represented mathematically. Therefore, when using a single SVM forecasting model, the forecast electricity MCP will lose most of its top and bottom peak values during the forecasting process. The proposed two-stage multiple SVM forecasting model can improve the compromise that is caused by using a single SVM by distributing the initially 
TABLE 8: Performance evaluation results between the proposed two-stage multiple SVM model and other forecasting models.

\begin{tabular}{|c|c|c|c|c|c|c|}
\hline & \multicolumn{3}{|c|}{ June 2009} & \multicolumn{3}{|c|}{ June 2010} \\
\hline & MAE & MAPE & MSRE & MAE & MAPE & MSRE \\
\hline Single SVM & 2.9743 & 11.7491 & 0.1564 & 7.2523 & 15.6454 & 0.3316 \\
\hline Single LSSVM & 2.8152 & 10.9722 & 0.1513 & 7.9003 & 16.2610 & 0.3964 \\
\hline Hybrid LSSVM and ARMAX & 2.7630 & 10.6706 & 0.1495 & 7.0989 & 13.9709 & 0.3859 \\
\hline Multiple LSSVM & 2.7811 & 10.7466 & 0.1463 & 7.0950 & 13.2850 & 0.3733 \\
\hline Two-stage multiple SVM & 2.9625 & 11.4083 & 0.1536 & 6.8106 & 14.6567 & 0.3126 \\
\hline
\end{tabular}

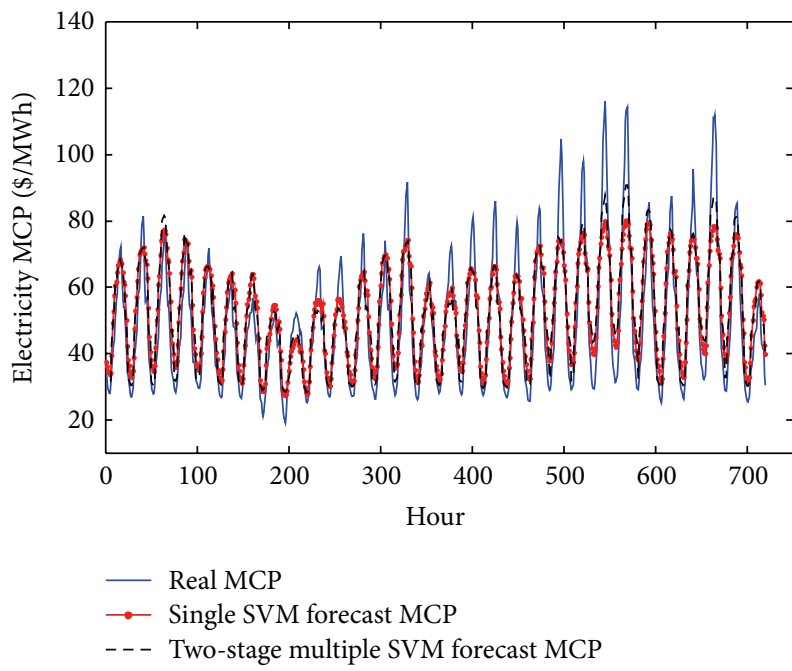

FIGURE 5: Forecasted electricity MCP in June 2010 using both single and two-stage multiple SVM.

predicted price values into different price zones. By doing so, instead of having only two control parameters in a single SVM forecasting model, the proposed two-stage multiple SVM forecasting model has 10 control parameters that can be adjusted to optimize the system forecasting accuracy.

\section{Conclusions}

A two-stage multiple SVM based midterm forecasting model of the electricity MCP is proposed in this paper. Two modules, input data distribution module and price forecasting module, are designed to first preprocess the initially predicted price values into corresponding price zones and then forecast the electricity price in four parallel designed SVMs. The proposed two-stage multiple SVM model showed improved forecasting accuracy in the low and the peak price zones and thus improving the overall forecasting accuracy compared to the forecasting model utilizing a single SVM. The case studies also show that the performance of either a single or a two-stage multiple SVM is highly depending on the selection of the input data. Carefully selected training input data and correctly predicted subdata sets would significantly improve the accuracy of the forecasting model. The proposed forecasting model has ten adjustable control parameters compared to only two adjustable control parameters in a single SVM forecasting model. The four parallel SVMs in the price forecasting module also have the flexibility of using different dimensions of input data to achieve the optimal results. Future research will focus on possible modules that can be added to the distribution module to improve the system accuracy.

\section{Conflict of Interests}

The authors declare that there is no conflict of interests regarding the publication of this paper.

\section{References}

[1] S. S. Torbaghan, A. Motamedi, H. Zareipour, and L. A. Tuan, "Medium-term electricity price forecasting," in Proceedings of the North American Power Symposium (NAPS '12), pp. 1-8, Champaign, Ill, USA, September 2012.

[2] X. Yan, Electricity market clearing price forecasting in a deregulated electricity market [M.S. dissertation], Department of Electrical and Computer Engineering, University of Saskatchewan, 2009.

[3] X. Yan and N. A. Chowdhury, "Electricity market clearing price forecasting in a deregulated electricity market," in Proceedings of the IEEE 11th International Conference on Probabilistic Methods Applied to Power Systems (PMAPS '10), pp. 36-41, IEEE, Singapore, June 2010.

[4] X. Yan and N. A. Chowdhury, Electricity Market Clearing Price Forecasting in a Deregulated Market: A Neural Network Approach, VDM, Saarbrücken, Germany, 2010.

[5] S. S. Torghaban, H. Zareipour, and L. A. Tuan, "Medium-term electricity market price forecasting: a data-driven approach," in Proceedings of the North American Power Symposium (NAPS '10), pp. 1-7, Arlington, Va, USA, September 2010.

[6] J. Contreras, R. Espínola, F. J. Nogales, and A. J. Conejo, "ARIMA models to predict next-day electricity prices," IEEE Transactions on Power Systems, vol. 18, no. 3, pp. 1014-1020, 2003.

[7] S. J. Yao and Y. H. Song, "Prediction of system marginal prices by wavelet transform and neural network," in Proceedings of the Electric Machines and Power Systems, pp. 983-993, 2000.

[8] C.-I. Kim, I.-K. Yu, and Y. H. Song, "Prediction of system marginal price of electricity using wavelet transform analysis," Energy Conversion and Management, vol. 43, no. 14, pp. 18391851, 2002.

[9] J. L. Zhang and Z. F. Tan, "Day-ahead electricity price forecasting using WT, CLSSVM and EGARCH model," International Journal of Electrical Power and Energy Systems, vol. 45, no. 1, pp. 362-368, 2013. 
[10] C. M. Ruibal and M. Mazumdar, "Forecasting the mean and the variance of electricity prices in deregulated markets," IEEE Transactions on Power Systems, vol. 23, no. 1, pp. 25-32, 2008.

[11] Z. Obradovic and K. Tomsovic, "Time series methods for forecasting electricity market pricing," in Proceedings of the IEEE Power Engineering Society Summer Meeting, vol. 2, pp. 1264-1265, Edmonton, Canada, 1999.

[12] J. Crespo Cuaresma, J. Hlouskova, S. Kossmeier, and M. Obersteiner, "Forecasting electricity spot-prices using linear univariate time-series models," Applied Energy, vol. 77, no. 1, pp. 87-106, 2004.

[13] F. J. Nogales, J. Contreras, A. J. Conejo, and R. Espínola, "Forecasting next-day electricity prices by time series models," IEEE Transactions on Power Systems, vol. 17, no. 2, pp. 342-348, 2002.

[14] F. Gao, X. H. Guan, X.-R. Cao, and A. Papalexpoulos, "Forecasting power market clearing price and quantity using a neural network method," in Proceedings of the IEEE Power Engineering Society Summer Meeting, vol. 4, pp. 2183-2188, Seattle, Wash, USA, July 2000.

[15] J. P. S. Catalão, S. J. P. S. Mariano, V. M. F. Mendes, and L. A. F. M. Ferreira, "Short-term electricity prices forecasting in a competitive market: a neural network approach," Electric Power Systems Research, vol. 77, no. 10, pp. 1297-1304, 2007.

[16] P. S. Georgilakis, "Market clearing price forecasting in deregulated electricity markets using adaptively trained neural networks," in Proceedings of the 4th Helenic Conference on Artificial Intelligence, pp. 56-66, Heraklion, Greece, May 2006.

[17] Z. Xu, Z. Y. Dong, and W. Liu, "Neural network models for electricity market forecasting," in Neural Networks Applications in Information Technology and Web Engineering, D. Wang and N. K. Lee, Eds., pp. 233-245, Borneo Publishing Corporation, Sarawak, Malaysia, 1st edition, 2005.

[18] D. Singhal and K. S. Swarup, "Electricity price forecasting using artificial neural networks," International Journal of Electrical Power and Energy Systems, vol. 33, no. 3, pp. 550-555, 2011.

[19] S. Anbazhagan and N. Kumarappan, "Day-ahead deregulated electricity market price classification using neural network input featured by DCT," International Journal of Electrical Power and Energy Systems, vol. 37, no. 1, pp. 103-109, 2012.

[20] W. Sun, J.-C. Lu, and M. Meng, "Application of time series based SVM model on next-day electricity price forecasting under deregulated power market," in Proceedings of the International Conference on Machine Learning and Cybernetics, pp. 23732378, August 2006.

[21] D. M. Zhou, F. Gao, and X. H. Guan, "Application of accurate online support vector regression in energy price forecast," in Proceedings of the 5th World Congress on Intelligent Control and Automation (WCICA '04), pp. 1838-1842, June 2004.

[22] L. L. Hu, G. Taylor, H. B. Wan, and M. Irving, "A review of shortterm electricity price forecasting techniques in deregulated electricity markets," in Proceedings of the 44th International Universities Power Engineering Conference, pp. 1-5, September 2009.

[23] R. A. Swief, Y. G. Hegazy, T. S. Abdel-Salam, and M. A. Bader, "Support vector machines (SVM) based short term electricity load-price forecasting," in Proceedings of the IEEE Bucharest PowerTech, pp. 1-5, Bucharest, Romania, June 2009.

[24] L. M. Saini, S. K. Aggarwal, and A. Kumar, "Parameter optimisation using genetic algorithm for support vector machinebased price-forecasting model in National electricity market,"
IET Generation, Transmission \& Distribution, vol. 4, no. 1, pp. 36-49, 2010.

[25] W. Sun and J. Zhang, "Forecasting day ahead spot electricity prices based on GASVM," in Proceedings of the International Conference on Internet Computing in Science and Engineering (ICICSE '08), pp. 73-78, Harbin, China, January 2008.

[26] Y.-G. Chen and G. W. Ma, "Electricity price forecasting based on support vector machine trained by genetic algorithm," in Proceedings of the 3rd International Symposium on Intelligent Information Technology Application (IITA '09), vol. 2, pp. 292295, November 2009.

[27] M. J. Mahjoob, M. Abdollahzade, and R. Zarringhalam, "GA based optimized LS-SVM forecasting of short term electricity price in competitive power markets," in Proceedings of the 3rd IEEE Conference on Industrial Electronics and Applications (ICIEA '08), pp. 73-78, June 2008.

[28] D.-S. Gong, J.-X. Che, J.-Z. Wang, and J.-Z. Liang, "Short-term electricity price forecasting based on novel SVM using artificial fish swarm algorithm under deregulated power," in Proceedings of the 2nd International Symposium on Intelligent Information Technology Application, pp. 85-89, December 2008.

[29] H. Zheng, L. Z. Zhang, L. Xie, and X. Li, "SVM model of system marginal price based on developed independent component analysis," in Proceedings of the International Conference on Power System Technology, pp. 1437-1440, 2004.

[30] Y. Wang and S. Q. Yu, "Price forecasting by ICA-SVM in the competitive electricity market," in Proceedings of the 3rd IEEE Conference on Industrial Electronics and Applications (ICIEA '08), pp. 314-319, Singapore, June 2008.

[31] J. Y. Tian and Y. Lin, "Short-term electricity price forecasting based on rough sets and improved SVM," in Proceedings of the 2nd International Workshop on Knowledge Discovery and Data Mining (WKKD '09), pp. 68-71, IEEE, Moscow, Russia, January 2009.

[32] T. Wang and L. Qin, "Application of SVM based on rough set in electricity prices forecasting," in Proceedings of the 2nd Conference on Environmental Science and Information Application Technology (ESIAT '10), pp. 317-320, IEEE, Wuhan, China, July 2010.

[33] L. Xie, H. Zheng, and L. Z. Zhang, "Electricity price forecasting by clustering-LSSVM," in Proceedings of the 8th International Power Engineering Conference, pp. 697-702, December 2007.

[34] S. Fan, J. R. Liao, K. Kaneko, and L. N. Chen, "An integrated machine learning model for day-ahead electricity price forecasting," in Proceedings of the IEEE PES Power Systems Conference and Exposition (PSCE '06), pp. 1643-1649, November 2006.

[35] S. Fan, C. Mao, and L. Chen, "Next-day electricity-price forecasting using a hybrid network," IET Generation, Transmission and Distribution, vol. 1, no. 1, pp. 176-182, 2007.

[36] J. H. Zhao, Z. Y. Dong, Z. Xu, and K. P. Wong, "A statistical approach for interval forecasting of the electricity price," IEEE Transactions on Power Systems, vol. 23, no. 2, pp. 267-276, 2008.

[37] J. H. Zhao, Z. Y. Dong, X. Li, and K. P. Wong, "A general method for electricity market price spike analysis," in Proceedings of the IEEE Power Engineering Society General Meeting, pp. 286-293, June 2005.

[38] J. H. Zhao, Z. Y. Dong, X. Li, and K. P. Wong, "A framework for electricity price spike analysis with advanced data mining methods," IEEE Transactions on Power Systems, vol. 22, no. 1, pp. 376-385, 2007. 
[39] P. Areekul, T. Senjyu, H. Toyama, and A. Yona, "A hybrid ARIMA and neural network model for short-term price forecasting in deregulated market," IEEE Transactions on Power Systems, vol. 25, no. 1, pp. 524-530, 2010.

[40] L. Xie, L. Z. Zhang, H. Zheng, and Y. Xie, "On the sensitivity analysis of electricity price," in Proceedings of the 2nd IEEE Conference on Industrial Electronics and Applications, pp. 16031606, May 2007.

[41] Y. Wang, "Price-load elasticity analysis by hybrid algorithm," in Proceedings of the $3 r$ International Conference on Electric Utility Deregulation and Restructuring and Power Technologies (DRPT '08), pp. 233-237, IEEE, Nanjing, China, April 2008.

[42] H. M. I. Pousinho, V. M. F. Mendes, and J. P. S. Catalão, "Shortterm electricity prices forecasting in a competitive market by a hybrid PSO-ANFIS approach," International Journal of Electrical Power and Energy Systems, vol. 39, no. 1, pp. 29-35, 2012.

[43] X. Yan and N. A. Chowdhury, "Mid-term electricity market clearing price forecasting: a hybrid LSSVM and ARMAX approach," International Journal of Electrical Power \& Energy Systems, vol. 53, no. 1, pp. 20-26, 2013.

[44] D. J. Pedregal and J. R. Trapero, "Mid-term hourly electricity forecasting based on a multi-rate approach," Energy Conversion and Management, vol. 51, no. 1, pp. 105-111, 2010.

[45] J. G. González, J. Barquín, and P. Dueñas, "A hybrid approach for modeling the electricity price series in the medium term," in Proceedings of the Power Systems Computation Conference (PSCC '08), 2008.

[46] C. Cortes and V. Vapnik, Support-Vector Networks, AT\&T Bell Labs, Holmdel, NJ, USA, 1995.

[47] Electricity LMP in PJM, 2014, http://www.pjm.com/marketsand-operations/energy/day-ahead/lmpda.aspx.

[48] Federal Energy Regulatory Commission, 2010, http://www.ferc .gov/. 


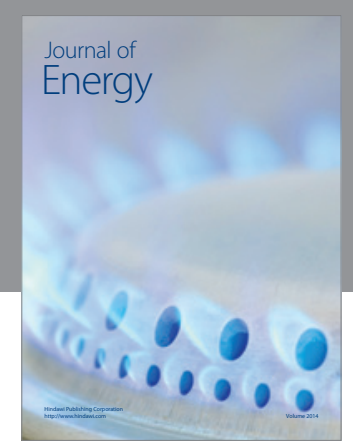

Journal of

Industrial Engineering
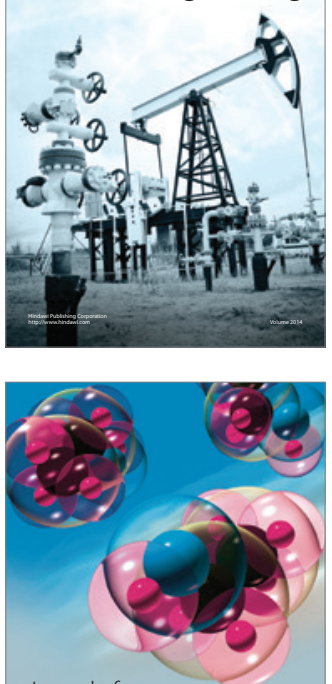

Fuels
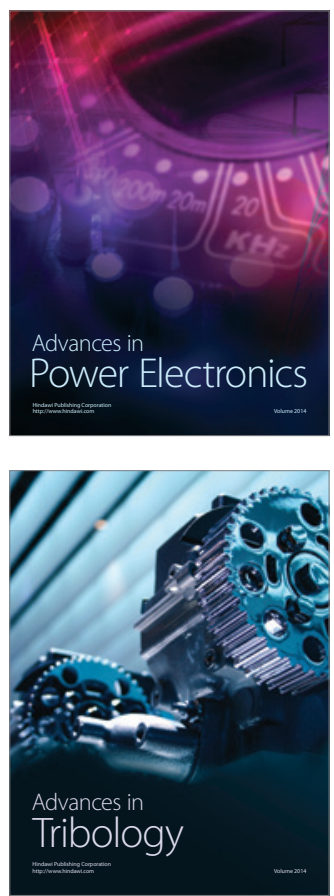

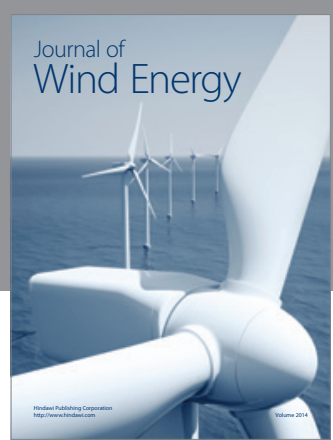

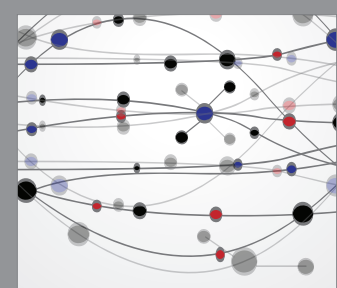

The Scientific World Journal

Submit your manuscripts at http://www.hindawi.com

Journal of

Structures
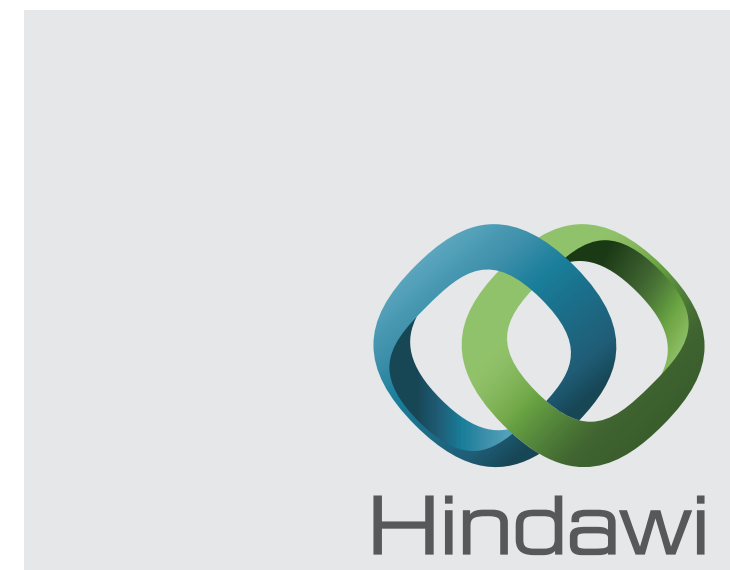

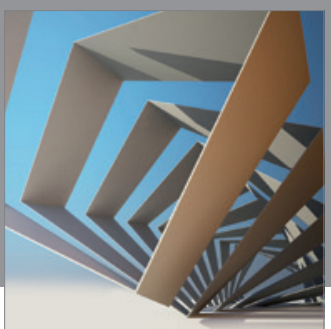

Rotating

Machinery
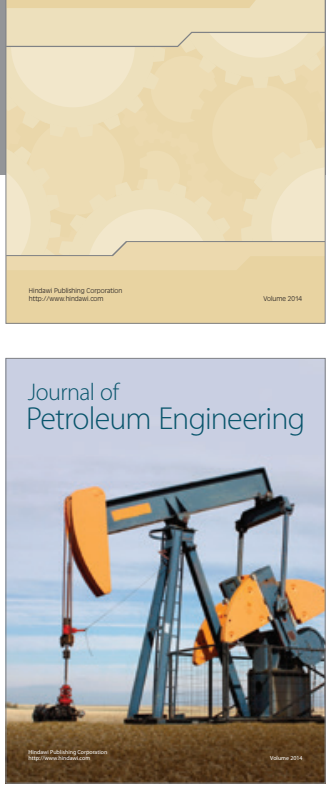

Journal of

Solar Energy
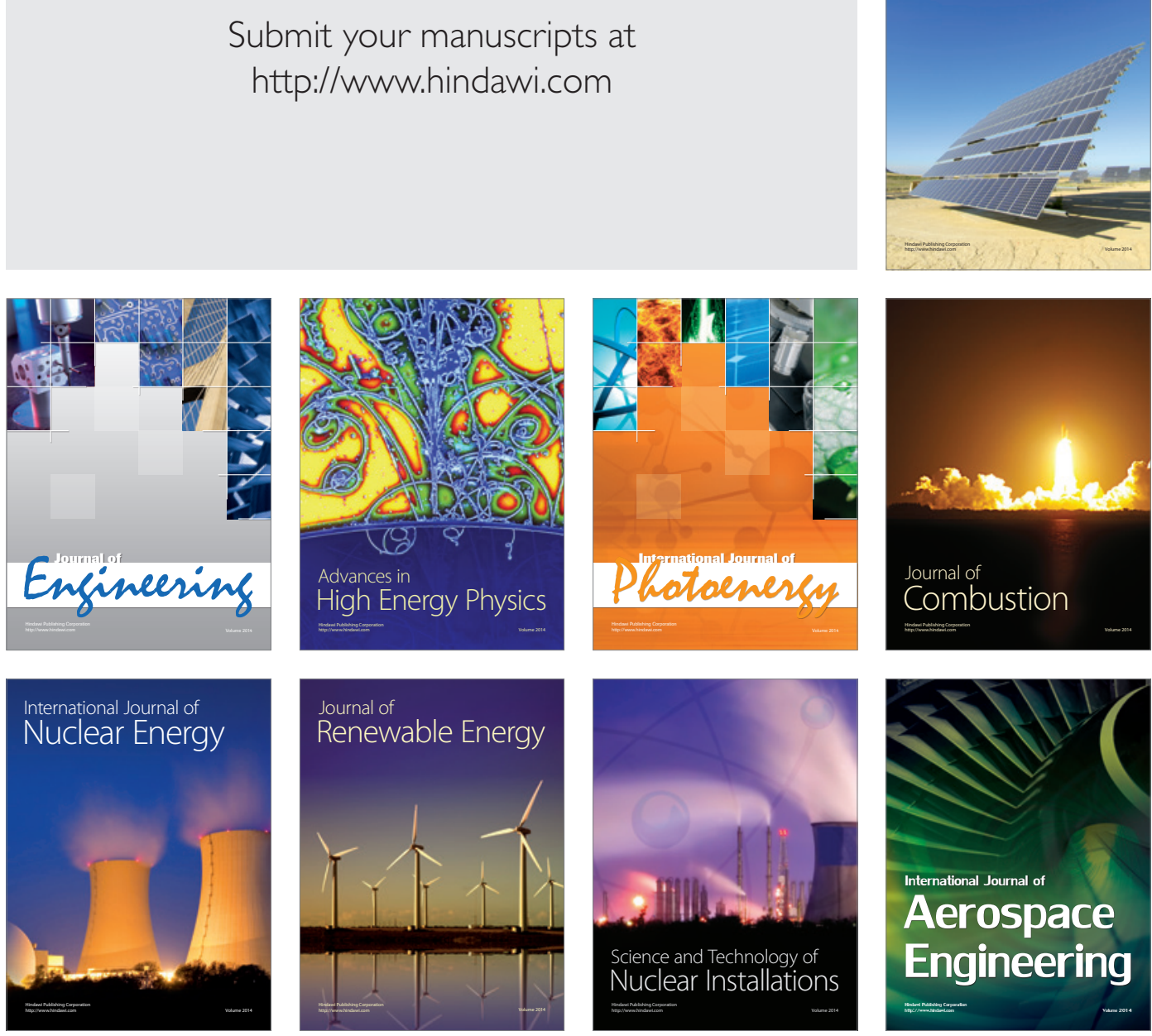